\title{
Polyhedral Potential and Variational Integrator Computation of the Full Two Body Problem
}

\author{
Eugene G. Fahnestock*, Taeyoung Lee, Melvin Leok ${ }^{\dagger}$ \\ N. Harris McClamroch $\ddagger$ and Daniel J. Scheeres ${ }^{\S}$ \\ University of Michigan, Ann Arbor, Michigan, USA
}

\begin{abstract}
We present a combination of tools which allows for investigation of the coupled orbital and rotational dynamics of two rigid bodies with nearly arbitrary shape and mass distribution, under the influence of their mutual gravitational potential. Methods for calculating that mutual potential and resulting forces and moments for a polyhedral body representation are simple and efficient. Discrete equations of motion, referred to as the Lie Group Variational Integrator (LGVI), preserve the structure of the configuration space, $\mathrm{SE}(3)$, as well as the geometric features represented by the total energy and the total angular momentum. The synthesis of these approaches allows us to simulate the full two body problem accurately and efficiently. Simulation results are given for two octahedral rigid bodies for comparison with other integration methods and to show the qualities of the results thus obtained. A significant improvement is seen over other integration methods while correctly capturing the interesting effects of strong orbit and attitude dynamics coupling, in multiple scenarios.
\end{abstract}

\section{Nomenclature}

E Total energy of system

$F \quad$ Update matrix, $\in \mathrm{SO}(3)$, for relative attitude rotation matrix $R$

$F_{2} \quad$ Update matrix, $\in \mathrm{SO}(3)$, for rotation matrix $R_{2}$

$G \quad$ Universal gravitational constant

$J_{1} \quad$ Standard moment of inertia matrix of $\mathcal{B}_{1}$ expressed in its own frame

$J_{2} \quad$ Standard moment of inertia matrix of $\mathcal{B}_{2}$ expressed in its own frame

$J_{R} \quad$ Standard moment of inertia matrix of $\mathcal{B}_{1}$ expressed in frame fixed to $\mathcal{B}_{2}$

$J_{d R} \quad$ Non-standard moment of inertia matrix of $\mathcal{B}_{1}$ expressed in frame fixed to $\mathcal{B}_{2}$

$J_{d_{1}} \quad$ Non-standard moment of inertia matrix of $\mathcal{B}_{1}$ expressed in its own frame

$J_{d_{2}} \quad$ Non-standard moment of inertia matrix of $\mathcal{B}_{2}$ expressed in its own frame

$K E \quad$ Kinetic energy of system

$L \quad$ Reduced Lagrangian for system

$M \quad$ Moment due to mutual gravitational potential

$N \quad$ Number of time-steps of length $h$ to go from the initial time to the final time in simulation

$P E \quad$ Potential energy of system

$R \quad$ Relative attitude rotation matrix, $\in \mathrm{SO}(3)$, mapping from the frame fixed to $\mathcal{B}_{1}$ to the frame fixed to $\mathcal{B}_{2}$

$R_{1} \quad$ Rotation matrix, $\in \mathrm{SO}(3)$, mapping from the frame fixed to $\mathcal{B}_{1}$ to the inertial reference frame

$R_{2} \quad$ Rotation matrix, $\in \mathrm{SO}(3)$, mapping from the frame fixed to $\mathcal{B}_{2}$ to the inertial reference frame

$S($.$) \quad Denotes the cross-product operation matrix.$

${ }^{*}$ Graduate Student, Department of Aerospace Engineering, University of Michigan, 2008 FXB Building, 1320 Beal Avenue, Ann Arbor, MI 48109, AIAA Student Member.

${ }^{\dagger}$ Assistant Professor, Department of Mathematics, University of Michigan.

¥Professor, Department of Aerospace Engineering, University of Michigan, AIAA Senior Member.

$\S$ Associate Professor, Department of Aerospace Engineering, University of Michigan, AIAA Associate Fellow. 
$T$ Jacobian determinant of the matrix containing coordinates of non-centroid vertices of a simplex

$U \quad$ Mutual gravitational potential

$\hat{U}_{\#} \quad$ The \#'th Legendre series term of increasing order within mutual gravitational potential equation

$V \quad$ Relative velocity vector of $\mathcal{B}_{1}$ with respect to $\mathcal{B}_{2}$ expressed in the frame fixed to $\mathcal{B}_{2}$

$V_{1} \quad$ Velocity vector of $\mathcal{B}_{1}$ 's centroid expressed in the frame fixed to $\mathcal{B}_{1}$

$V_{2} \quad$ Velocity vector of $\mathcal{B}_{2}$ 's centroid expressed in the frame fixed to $\mathcal{B}_{2}$

$X \quad$ Relative position vector between body centroids expressed in the frame fixed to $\mathcal{B}_{2}$

$X_{1} \quad$ Position vector of $\mathcal{B}_{1}$ 's centroid expressed in the frame fixed to $\mathcal{B}_{1}$

$X_{2} \quad$ Position vector of $\mathcal{B}_{2}$ 's centroid expressed in the frame fixed to $\mathcal{B}_{2}$

$\mathcal{B}_{1}, \mathcal{B}_{2}$ Labels for the two polyhedral rigid bodies

$\Omega \quad$ Angular velocity vector of $\mathcal{B}_{1}$ expressed in the frame fixed to $\mathcal{B}_{2}$

$\Omega_{1} \quad$ Angular velocity vector of $\mathcal{B}_{1}$ expressed in the frame fixed to $\mathcal{B}_{1}$

$\Omega_{2} \quad$ Angular velocity vector of $\mathcal{B}_{2}$ expressed in the frame fixed to $\mathcal{B}_{2}$

$\delta \quad$ Rank 2 tensor defined by the Kronecker delta function

$\gamma_{T} \quad$ Total linear momentum of system expressed in inertial reference frame

$\pi_{T} \quad$ Total angular momentum of system expressed in inertial reference frame

$\rho \quad$ Density, $\mathrm{kg} / \mathrm{m}^{3}$

a Simplex in $\mathcal{B}_{1}$

b Simplex in $\mathcal{B}_{2}$

$h \quad$ Integration step size

$m \quad$ Scalar mass parameter for the system

$m_{1} \quad$ Mass of body $\mathcal{B}_{1}$

$m_{2} \quad$ Mass of body $\mathcal{B}_{2}$

$r \quad$ Scalar magnitude of the relative position vector between body centroids, $r=\|X\|$

$t \quad$ Simulation time

$t_{0} \quad$ Time at start of simulation

$t_{f} \quad$ Time at end of simulation

$v_{1} \quad$ Velocity vector of $\mathcal{B}_{1}$ 's centroid expressed in the inertial reference frame

$v_{2} \quad$ Velocity vector of $\mathcal{B}_{2}$ 's centroid expressed in the inertial reference frame

$x_{1} \quad$ Position vector of $\mathcal{B}_{1}$ 's centroid expressed in the inertial reference frame

$x_{2} \quad$ Position vector of $\mathcal{B}_{2}$ 's centroid expressed in the inertial reference frame

D Rank 4 tensor, $\in \mathbb{R}^{3 \times 6 \times 3 \times 3}$, the partial derivative of $\mathbf{v}$ with respect to a rotation matrix

Q Tensor that is symmetric along every dimension, in which each element is a rational number, with form illustrated in Ref. 1

$\mathbf{r} \quad$ Rank 2 tensor, $\in \mathbb{R}^{6 \times 6}$, dependent on relative attitude but not relative position

$\mathbf{v} \quad$ Rank 2 tensor, $\in \mathbb{R}^{3 \times 6}$, containing coordinates of non-centroid vertices of both simplices in a simplex pairing, expressed in any desired coordinate frame

w $\quad$ Rank 1 tensor, $\in \mathbb{R}^{6}$, dependent on relative attitude and relative position

\section{Subscripts}

$\phi \theta \quad$ Tensor indices not eliminated by summation

a Denotes "for simplex $a$ in $\mathcal{B}_{1}$ "

$b \quad$ Denotes "for simplex $b$ in $\mathcal{B}_{2}$ "

$c 1 c 2$ c3 Denotes the first, second, third column vectors of a matrix, respectively

$i j k p$ Tensor indices eliminated by summation in left hand side of equations

$n \quad$ Second level subscript, denotes the value of variables at simulation time $t=n h+t_{0}$

Superscripts

$\phi \theta \quad$ Tensor indices not eliminated by summation

$i j k p$ Tensor indices eliminated by summation in left hand side of equations

$T \quad$ Denotes matrix transpose

\section{Introduction}

$T^{\text {HE }}$ full two body problem studies the dynamics of two irregular rigid bodies interacting under a mutual 1 potential. The full two body problem arises in numerous engineering and scientific fields. Our focus is on 
the dynamics of two rigid bodies in space due to their mutual gravitational potential. This depends on both the relative position and the relative attitude of the bodies. Therefore, the translational orbit dynamics and the rotational attitude dynamics are coupled in the full two body problem. For example, the trajectory of a very large spacecraft around the Earth is affected by the attitude of the spacecraft, and the dynamics of a binary asteroid pair are characterized by the non-spherical mass distributions of the two bodies. Recently, interest in the full (two) body problem as applied to binary objects in space has increased, due to evidence that such binary systems are common among the overall asteroid population and form an especially large percentage of near-earth objects, with some estimates as high as 16 percent. $^{2-5}$ In addition, advances in radar and optical observation methods ${ }^{6}$ have allowed for better modelling of the shapes and characteristics of bodies in binary systems, encouraging further study of their dynamics.

Some previous work of relevance includes Maciejewski's presentation of equations of motion of the full two body problem in inertial and relative coordinates. ${ }^{7}$ He also discussed the existence of relative equilibria. Scheeres derived a stability condition for the full two body problem, ${ }^{8}$ and he studied the planar stability of an ellipsoid-sphere model. ${ }^{9}$ Spacecraft motion about binary asteroids has been discussed using the restricted three body model, ${ }^{10,11}$ and the four body model. ${ }^{12}$

The mutual gravitational potential of two rigid celestial bodies has been expressed using spherical harmonics. ${ }^{13,14}$ But, the harmonic expansion is not guaranteed to converge. Convergence is shown to be an unstable property of such spherical harmonic series. ${ }^{15}$ By this we mean that an arbitrarily small change to the mass distribution may cause a previously convergent series to diverge. Another commonly used approach for evaluating the mutual gravitational potential is to fill each rigid body's volume with a distribution of point masses, fixed with respect to one another, the sum of which equals the respective body's total mass. ${ }^{16,17}$ Although the mutual potential obtained for two rigid bodies using this approach converges to the true gravity field in the limit as the number of point masses becomes arbitrarily large, there are significant errors in the computation of gravitational forces from that mutual potential. ${ }^{18}$

These problems are avoided with the approach presented in Ref. 1 for calculating mutual gravitational potential based on polyhedral body models. This method converges at all points exterior to the two bodies. By representing the bodies as polyhedra, the flexibility of the formalism over more specialized representations such as spheres and ellipsoids is increased. A polyhedral rigid body model can directly include important features such as craters, caves, or deep clefts where contact binaries meet. The entire body does not have to be modelled uniformly at a high resolution. The errors in the mutual potential computation can be reduced to the level of error in each body's shape determination, and to the level of discretization chosen for that shape. Derivatives of this polyhedral mutual potential formulation are given in Ref. 19 and, with some corrections and improvement of notation, in Ref. 20. These derivatives determine the forces and torques exerted by the bodies on each other, for use in either inertial or relative equations of motion that describe the full body dynamics.

These full body dynamics arise from Lagrangian and Hamiltonian mechanics; they are characterized by symplectic, momentum and energy preserving properties. These geometric features determine the qualitative behavior of the full body dynamics, and they can serve as a basis for further theoretical study of the full body problem. The configuration space of the full body dynamics have a Lie group structure referred to as the Euclidean group, $\mathrm{SE}(3)$. However, general numerical integration methods, including the widely used Runge-Kutta schemes, neither preserve the Lie group structure nor these geometric properties. ${ }^{21}$

The variational approach ${ }^{22}$ and Lie group methods ${ }^{23}$ provide systematic methods of constructing structure preserving numerical integrators. The idea of the variational approach is to discretize Hamilton's principle rather than the continuous equations of motion. ${ }^{22}$ The numerical integrator obtained from the discrete Hamilton's principle exhibits excellent energy properties, conserves first integrals, and preserves the symplectic structure. Lie group methods consist of numerical integrators that preserve the geometry of the configuration space by automatically remaining on the Lie group. ${ }^{23}$ A Lie group method is explicitly adopted for the variational integrator in Ref. 24 and 25. This unified integrator, hereafter referred to as the Lie Group Variational Integrator (or LGVI for short), is symplectic and momentum preserving, and it exhibits good total energy behavior for exponentially long time periods. It also preserves the Euclidian Lie group structure without the use of local charts, reprojection, or constraints.

Numerical simulation of the full body problem involves two major problems; a large computational burden in computing mutual gravitational forces and moments, and inaccuracy of numerical integrators. The forces and moments must constantly be reevaluated with any position change or any orientation change, not only at each time step but at each sub-step involved in the differencing scheme behind any general 
numerical integrator. Therefore such general numerical integrators amplify the computation cost for finding the forces and moments. The accuracy of such general integrators also rapidly degrades as the simulation time increases. They fail to preserve the conserved quantities such as total energy and angular momentum, which determine the qualitative behavior of the full body dynamics. Attitude errors tend to accumulate as a consequence of numerical errors, and this attitude degradation causes significant errors in the gravitational force and moment computation.

The unified treatment given in this paper of the polyhedral mutual potential formulation combined with the LGVI presents a solution to these two major dynamic simulation problems. Using polyhedral models, we can approximate irregular bodies to a specified accuracy, and we can control the computational burden to compute the mutual gravitational forces and moments by choosing the level of body discretization and the number of series terms employed in our formulation. The LGVI, as presented in this paper, preserves the conserved quantities of the full body dynamics as well as the orthogonal structure of the rotation matrices. The obtained simulation results exhibit good stability properties for the invariants of motion for exponentially long times. The computational load is further minimized since the LGVI requires one force and torque evaluation per integration step for second order accuracy.

Subsequent sections of this paper are organized as follows. Algorithms for the mutual gravitational forces and moments computation for polyhedral body models are presented in section II. Some description of the full two body problem and its mathematical properties and the continuous relative equations of motion are given in section III. The Lie Group Variational Integrator for computing the dynamics of the full body problem is given in section IV. Selected simulation results are given in section V for two octahedral rigid bodies in several scenarios, for comparison with other integration methods and to show the qualities of the results thus obtained. Conclusions drawn from the results for these scenarios about the accuracy and suitability of our methods, and about computational burden, are given in the last section.

\section{Polyhedral Mutual Gravitational Potential, Forces, and Moments}

In this section, we present computational algorithms for determining the mutual gravitational potential, forces, and moments given polyhedral models of each of the two rigid bodies. The algorithm to compute the mutual potential relatively efficiently with such a modelling approach is outlined in Ref. 1 . The methods outlined in Ref. 19, and in Ref. 20 with some corrections and improvement of notation, can then be used to compute the gradients of the mutual potential (i.e. forces and moments). A more detailed description and derivation of materials in this section, including extensions to computing the forces and moments for inertial equations of motion, can be found in Ref. 20.

\section{A. Context for Methodology}

In Ref. 1, a uniform density or homogenous mass distribution within the entire volume of each rigid body is assumed. This may be a realistic assumption for binary asteroids based on some empirical data. ${ }^{26}$ However, the underlying method does also allow for approximating arbitrary density variations, as required for greater asteroid modelling fidelity and for systems in which one or more bodies is a spacecraft. This is mentioned without proper explanation in Ref. 20, so we will be more specific here. Rather than representing each body with just a single polyhedron whose triangular faces form the body's outer surface, as in prior work, we can represent each body by multiple polyhedra partially or fully nested inside of one another. Each polyhedron has a closed surface consisting of triangular faces, and each triangular face is defined by three vertices. A tetrahedron is formed by these three vertices plus the body centroid. This tetrahedron is referred to hereafter as a simplex. We require each simplex to have a constant density, but different densities can be assigned to each simplex. This allows for density variation over the two angular spherical coordinates within each polyhedron, with resolution determined by the size of the faces. The possible nesting of multiple polyhedra allows for density variation over the radial spherical coordinate within the body volume as well. It should be noted though that to make practical use of the method herein, the coordinates with respect to the body centroid of the three non-centroid vertices of each simplex must be known. While this vertex coordinate information is known throughout for spacecraft bodies, and can be derived for exterior faces of natural bodies from shape data obtained via optical and radar observations or LIDAR surveying, it is difficult to specify for interior faces within natural bodies without specific knowledge of internal mass distribution.

The underlying principle of what follows is that the evaluation of the mutual potential's double volume 
integral over both bodies is equivalent to a global sum of the results of evaluating that double volume integral over each possible pairing of simplices, one being drawn from each body. For each such pairing, the contribution to the mutual potential is given by a Legendre-series expansion. Successive terms of this expansion are linear combinations of other terms which factor into symmetric tensors of increasing rank that are independent of relative position and attitude, and other tensors that depend on the relative position and attitude. The gradients of the mutual potential according to this formulation make use of efficient tensor differentiation rules and the chain rule, resulting in a series expansion for the forces and moments that converges for all points exterior to all polyhedra representing the bodies, and hence for all points exterior to the bodies.

It is useful to note here that the number of terms kept in the Legendre-series expansion determines the order, in the inverse of the distance between body centroids, of the errors in the forces and the moments. When the rigid bodies have small separation distances, more terms are required to maintain the same error levels. A possibility for adaptivity during simulation is to adjust the number of terms used in the series expansion for the force and moment computations, depending on the body separation distance. Another possibility is to refine one or more of the polyhedra representing each body as separation distance decreases. However, this must be done carefully, in a manner which involves no change to the relevant conserved quantities and no discontinuity in the motion states.

It is also worth mentioning that one can consider our approach as a special case of a more general problem, not treated in this paper, in which the two bodies are no longer rigid but fragmented, i.e. they are so-called "rubble-piles". The rubble-pile model of asteroids is being supported by an increasing body of evidence from recent asteroid exploration missions. ${ }^{27} \mathrm{~A}$ number of researchers have represented such rubble-pile asteroids as collections of nonintersecting spheres held together under self-gravity, ${ }^{28}$ not to be confused with representing a rigid body by filling its volume with spheres fixed with respect to one another. One could just as well represent rubble-pile asteroids as collections of ellipsoids or polyhedra of arbitrary size and shape held together under self-gravity, rather than smooth spheres, as in Refs. 29 and 30 respectively. This allows for filling a body's volume more fully or at different porosities than are possible with spheres (even zero initial porosity with polyhedra). With polyhedra, different levels of rigidity within a body can then be considered simply by initial combination of smaller polyhedra with coincident or adjacent faces into larger polyhedra. The full two rigid body problem that is the subject of this paper can then be viewed from a different perspective as the limiting case of that process of combination within two separated bodies. This is similar to using a tree-code method, ${ }^{31}$ but only using the root- or top-level cell (or group) within each body. In other cases employing multiple non-intersecting polyhedra within each body, the gravitational force and moment couples between every possible pair of polyhedra in the binary system can still be accurately evaluated using the methodology of this section, considered independently from the rest of this paper.

\section{B. Mutual Gravitational Potential}

We label the two polyhedral rigid bodies $\mathcal{B}_{1}$ and $\mathcal{B}_{2}$. Consistent with the earlier discussion and definition of the polyhedral modelling approach, let body $\mathcal{B}_{1}$ be divided into a set of simplices indexed by $a$ and let body $\mathcal{B}_{2}$ be divided into a set of simplices indexed by $b$. Evaluating the double volume integrals over $\mathcal{B}_{1}$ and $\mathcal{B}_{2}$ is equivalent to the double summation over all $a$ and over all $b$ of the result of evaluating the double volume integrals over each simplex combination $(a, b)$. This is shown in the following expression for the mutual potential. ${ }^{1}$ Note that at this point, we make use of tensor notation and the Einstein convention of summation over repeated indices:

$$
\begin{aligned}
U=-G \sum_{a \in \mathcal{B}_{1}} \sum_{b \in \mathcal{B}_{2}} \rho_{a} T_{a} \rho_{b} T_{b}\left\{\left[\frac{\mathbf{Q}}{r}\right]+\left[-\frac{\mathbf{Q}_{i} \mathbf{w}^{i}}{r^{3}}\right]+\left[-\frac{\mathbf{Q}_{i j} \mathbf{r}^{i j}}{2 r^{3}}+\frac{3 \mathbf{Q}_{i j} \mathbf{w}^{i} \mathbf{w}^{j}}{2 r^{5}}\right]\right. \\
\left.+\left[\frac{3 \mathbf{Q}_{i j k} \mathbf{r}^{i j} \mathbf{w}^{k}}{2 r^{5}}-\frac{5 \mathbf{Q}_{i j k} \mathbf{w}^{i} \mathbf{w}^{j} \mathbf{w}^{k}}{2 r^{7}}\right]+\ldots\right\}
\end{aligned}
$$

The scalars $T_{a}$ and $T_{b}$ and the tensors $\mathbf{Q}$ of increasing rank are all independent of both relative position between centroids and relative attitude between the bodies, so they can be computed before any dynamic simulation. However the vector $\mathbf{w}$ is dependent on relative position, and both the vector $\mathbf{w}$ and the matrix $\mathbf{r}$ are dependent on relative attitude through a matrix $\mathbf{v}$, where

$$
\mathbf{w}^{i}=\mathbf{v}_{j}^{i} X^{j} \quad, \quad \mathbf{r}^{i j}=\mathbf{v}_{p}^{i} \mathbf{v}_{p}^{j} .
$$


The Q's are defined in Ref. 1, in which they are written out explicitly up to the third rank for illustration of their form. The series within the braces in Eq. (1) is infinite but sufficient accuracy seems to be obtained with just the first several terms in square brackets. We denote these bracketed terms as scalars $\hat{U}_{0}, \hat{U}_{1}, \hat{U}_{2}$, and so on.

\section{Mutual Gravitational Forces and Moments}

The gravitational force existing between the two bodies is given by the partial derivative of the mutual potential with respect to the relative position vector $X$. To obtain this we first derive simple tensor differentiation rules: ${ }^{20}$

$$
\frac{\partial r}{\partial X_{\theta}}=\frac{X_{\theta}}{r} \quad, \quad \frac{\partial \mathbf{w}^{i}}{\partial X_{\theta}}=\mathbf{v}_{\theta}^{i}
$$

These are used in finding each successive $\partial \hat{U} / \partial X_{\theta}$ term. The first few of these terms are illustrated below:

$$
\begin{aligned}
& \frac{\partial \hat{U}_{0}}{\partial X_{\theta}}=-\frac{\mathbf{Q} X_{\theta}}{r^{3}}, \\
& \frac{\partial \hat{U}_{1}}{\partial X_{\theta}}=\frac{3 \mathbf{Q}_{i} X_{\theta} \mathbf{w}^{i}}{r^{5}}-\frac{\mathbf{Q}_{i} \mathbf{v}_{\theta}^{i}}{r^{3}}, \\
& \frac{\partial \hat{U}_{2}}{\partial X_{\theta}}=\frac{3 \mathbf{Q}_{i j} \mathbf{r}^{i j} X_{\theta}}{2 r^{5}}-\frac{15 \mathbf{Q}_{i j} X_{\theta} \mathbf{w}^{i} \mathbf{w}^{j}}{2 r^{7}}+\frac{3 \mathbf{Q}_{i j} \mathbf{w}^{i} \mathbf{v}_{\theta}^{j}}{r^{5}}, \\
& \frac{\partial \hat{U}_{3}}{\partial X_{\theta}}=-\frac{15 \mathbf{Q}_{i j k} \mathbf{r}^{i j} X_{\theta} \mathbf{w}^{k}}{2 r^{7}}+\frac{3 \mathbf{Q}_{i j k} \mathbf{r}^{i j} \mathbf{v}_{\theta}^{k}}{2 r^{5}}+\frac{35 \mathbf{Q}_{i j k} X_{\theta} \mathbf{w}^{i} \mathbf{w}^{j} \mathbf{w}^{k}}{2 r^{9}}-\frac{15 \mathbf{Q}_{i j k} \mathbf{w}^{i} \mathbf{w}^{j} \mathbf{v}_{\theta}^{k}}{2 r^{7}} .
\end{aligned}
$$

Such terms are used in the overall expression for the force vector that is in turn used in the relative equations of motion, namely (laying aside the tensor notation for the moment)

$$
\frac{\partial U}{\partial X}=-G \sum_{a \in \mathcal{B}_{1}} \sum_{b \in \mathcal{B}_{2}} \rho_{a} T_{a} \rho_{b} T_{b}\left(\frac{\partial \hat{U}_{0}}{\partial X}+\frac{\partial \hat{U}_{1}}{\partial X}+\frac{\partial \hat{U}_{2}}{\partial X}+\cdots\right) .
$$

The torque or moment between the bodies due to their mutual gravitational interaction is given by an expression requiring the partial derivative of the mutual potential with respect to the relative attitude $R$. Evaluating this requires partial differentiation of one attitude rotation matrix, an element of $\mathrm{SO}(3)$, with respect to another such matrix. For this we use the basic rule

$$
\frac{\partial R^{j k}}{\partial R^{\phi \theta}}=\delta_{j}^{\phi} \delta_{\theta}^{k}
$$

inside of the expression for the tensor $\mathbf{D}$. The details of this tensor and an alternate rule are discussed in Ref. 20. It is in turn used in the two tensor differentiation rules: ${ }^{20}$

$$
\frac{\partial \mathbf{w}^{i}}{\partial R^{\phi \theta}}=X^{j} \mathbf{D}_{j \theta}^{\phi i} \quad, \quad \frac{\partial \mathbf{r}^{i j}}{\partial R^{\phi \theta}}=2 \mathbf{v}_{p}^{i} \mathbf{D}_{p \theta}^{\phi j}
$$

These are used in finding each successive $\partial \hat{U} / \partial R^{\phi \theta}$ term. There is no attitude dependence in $\hat{U}_{0}$, and then the next few partials are:

$$
\begin{aligned}
\frac{\partial \hat{U}_{1}}{\partial R^{\phi \theta}} & =-\frac{\mathbf{Q}_{i} X^{j} \mathbf{D}_{j \theta}^{\phi i}}{r^{3}}, \\
\frac{\partial \hat{U}_{2}}{\partial R^{\phi \theta}} & =-\frac{\mathbf{Q}_{i j} \mathbf{v}_{p}^{i} \mathbf{D}_{p \theta}^{\phi j}}{r^{3}}+\frac{3 \mathbf{Q}_{i j} \mathbf{w}^{i} X^{p} \mathbf{D}_{p \theta}^{\phi j}}{r^{5}}, \\
\frac{\partial \hat{U}_{3}}{\partial R^{\phi \theta}} & =\frac{3 \mathbf{Q}_{i j k}}{2 r^{5}}\left(2 \mathbf{v}_{p}^{i} \mathbf{D}_{p \theta}^{\phi j} \mathbf{w}^{k}+\mathbf{r}^{i j} X^{p} \mathbf{D}_{p \theta}^{\phi k}\right)-\frac{15 \mathbf{Q}_{i j k} \mathbf{w}^{i} \mathbf{w}^{j} X^{p} \mathbf{D}_{p \theta}^{\phi k}}{2 r^{7}} .
\end{aligned}
$$

Such terms are used in the overall expression

$$
\frac{\partial U}{\partial R^{\phi \theta}}=-G \sum_{a \in \mathcal{B}_{1}} \sum_{b \in \mathcal{B}_{2}} \rho_{a} T_{a} \rho_{b} T_{b}\left(\frac{\partial \hat{U}_{1}}{\partial R^{\phi \theta}}+\frac{\partial \hat{U}_{2}}{\partial R^{\phi \theta}}+\frac{\partial \hat{U}_{3}}{\partial R^{\phi \theta}}+\ldots\right) .
$$

Using this within Eq. (17) below, we can evaluate the moment due to the mutual gravitational potential. 


\section{Full Two Body Dynamics and Continuous Equations of Motion}

In this section, we describe the full two rigid body problem, and we present the continuous relative equations of motion (EOM) and the conserved quantities for the full two rigid body dynamics. Continuous EOM for the full two rigid body problem are given in Ref. 7, and they are formally derived in the context of Lagrangian mechanics in Ref. 25. A more detailed description and derivation of materials in this section, including derivation of inertial EOM in addition to relative EOM, can be found in the latter reference.

The physical configuration of the full two rigid body problem is described in terms of the position vector of each rigid body in an inertial reference frame, an element of $\mathbb{R}^{3}$, and the attitude of each rigid body with respect to that frame. This attitude is represented by a rotation matrix, which is a $3 \times 3$ orthogonal matrix with determinant +1 , and hence is an element of the Lie group $\mathrm{SO}(3)$. The configuration space of each rigid body is therefore a Lie group referred to as the Euclidean Lie group, $\left.\operatorname{SE}(3)=\mathbb{R}^{3} \mathrm{~S}\right) \mathrm{SO}(3)$ (with $(\mathrm{S})$ meaning the semi-direct product). The motion of the two rigid bodies depends only on the relative positions and the relative attitudes of the bodies. This is a consequence of the property that the mutual gravitational potential depends only on these relative variables. Thus, the Lagrangian of the two rigid bodies is invariant under a group action of $\mathrm{SE}(3)$, and it is natural to reduce the EOM for the full body problem by writing them in one of the body fixed frames. Without loss of generality, we present the EOM in the frame fixed to $\mathcal{B}_{2}$. Then the configuration space of the reduced system is $\mathrm{SE}(3)$.

We start with the relative variables

$$
\begin{aligned}
X & =R_{2}^{T}\left(x_{1}-x_{2}\right), \\
R & =R_{2}^{T} R_{1},
\end{aligned}
$$

where $X \in \mathbb{R}^{3}$ is the relative position of $\mathcal{B}_{1}$ with respect to $\mathcal{B}_{2}$ expressed in the frame fixed to $\mathcal{B}_{2}$, and $R \in \mathrm{SO}(3)$ is the relative attitude of $\mathcal{B}_{1}$ with respect to $\mathcal{B}_{2}$. The corresponding linear and angular velocities are

$$
\begin{aligned}
& V=R_{2}^{T}\left(v_{1}-v_{2}\right), \\
& \Omega=R \Omega_{1},
\end{aligned}
$$

where $V \in \mathbb{R}^{3}$ represents the relative velocity of $\mathcal{B}_{1}$ with respect to $\mathcal{B}_{2}$ expressed in the frame fixed to $\mathcal{B}_{2}$, and $\Omega \in \mathbb{R}^{3}$ is the angular velocity of $\mathcal{B}_{1}$ expressed in the frame fixed to $\mathcal{B}_{2}$. The standard and nonstandard moment of inertia matrices (see Ref. 25 for the distinction) of $\mathcal{B}_{1}$ can also be expressed in the frame fixed to $\mathcal{B}_{2}$ according to $J_{R}=R J_{1} R^{T}$ and $J_{d R}=R J_{d_{1}} R^{T}$. Note that $J_{R}$ and $J_{d R}$ are not constant matrices.

Assuming that the total linear momentum of the whole system is zero, the kinetic energy of the system is expressed in terms of the relative variables as

$$
K E=\frac{1}{2} m\|V\|^{2}+\frac{1}{2} \operatorname{tr}\left[S(\Omega) J_{d R} S(\Omega)^{T}\right]+\frac{1}{2} \operatorname{tr}\left[S\left(\Omega_{2}\right) J_{d_{2}} S\left(\Omega_{2}\right)^{T}\right],
$$

where $m=\frac{m_{1} m_{2}}{m_{1}+m_{2}} \in \mathbb{R}$, and $S(\cdot): \mathbb{R}^{3} \mapsto \mathfrak{s o}(3)$ is an isomorphism between $\mathbb{R}^{3}$ and skew-symmetric matrices, defined such that $S(x) y=x \times y$ for any $x, y \in \mathbb{R}^{3}$. We also have from Eq. (1) the potential energy expressed in terms of the relative variables, that is to say $P E=U(X, R)$, recalling that in Eq. (1) $r$ and $\mathbf{w}$ are functions of $X$ and both $\mathbf{w}$ and $\mathbf{r}$ are functions of $R$. The following continuous EOM of the full two rigid body problem in relative coordinates can be obtained by taking variations of the reduced Lagrangian, $L=K E-P E$, as follows:

$$
\begin{gathered}
\dot{X}+\Omega_{2} \times X=V, \\
\dot{R}=S(\Omega) R-S\left(\Omega_{2}\right) R, \\
m \dot{V}+m \Omega_{2} \times V=-\frac{\partial U}{\partial X}, \\
\left(J_{R} \Omega\right)+\Omega_{2} \times J_{R} \Omega=-M, \\
J_{2} \dot{\Omega}_{2}+\Omega_{2} \times J_{2} \Omega_{2}=X \times \frac{\partial U}{\partial X}+M,
\end{gathered}
$$


where the vector $J_{R} \Omega=R J_{1} \Omega_{1} \in \mathbb{R}^{3}$ is the angular momentum of the first body expressed in the second body fixed frame. The moment due to the gravity potential, $M \in \mathbb{R}^{3}$, is determined by the following relationship

$$
S(M)=\frac{\partial U}{\partial R} R^{T}-R \frac{\partial U^{T}}{\partial R}
$$

or more explicitly,

$$
M=R_{c 1} \times\left[\frac{\partial U}{\partial R}\right]_{c 1}+R_{c 2} \times\left[\frac{\partial U}{\partial R}\right]_{c 2}+R_{c 3} \times\left[\frac{\partial U}{\partial R}\right]_{c 3} .
$$

These equations are also given in different notation in Ref. 20. The total energy, the total linear momentum expressed in the inertial frame, and the total angular momentum expressed in the inertial frame are conserved quantities, given by

$$
\begin{aligned}
E & =K E+P E \\
\gamma_{T} & =m_{1} v_{1}+m_{2} v_{2}, \\
\pi_{T} & =x_{1} \times m_{1} v_{1}+R_{1} J_{1} \Omega_{1}+x_{2} \times m_{2} v_{2}+R_{2} J_{2} \Omega_{2} .
\end{aligned}
$$

\section{Discrete Equations of Motion: the Lie Group Variational Integrator}

This section presents the LGVI, essentially a statement of discrete relative EOM in Hamiltonian form, as opposed to continuous relative EOM in Lagrangian form in the previous section. Again, a detailed description and derivation of materials in this section, including development of inertial discrete EOM in addition to these relative discrete EOM, can be found in Ref. 25. Here we simply state the results.

\section{A. Discrete Equations of Motion}

Since the dynamics of the full two rigid bodies arise from Lagrangian or Hamiltonian mechanics, they are characterized by symplectic, momentum and energy preserving properties. These geometric features determine the qualitative behavior of the dynamics, and they can serve as a basis for further theoretical study of the full two rigid body problem. Furthermore, the configuration space has a group structure denoted by $\mathrm{SE}(3)$.

However, general numerical integration methods, including the popular Runge-Kutta methods, fail to preserve these geometric characteristics. General integration methods are obtained by approximating continuous EOM by directly discretizing them with respect to time. With each integration step, the updates involve additive operations, so that the underlying Lie group structure is not necessarily preserved as time progresses. This is caused by the fact that the Euclidean Lie group is not closed under addition.

For example, if we use a Runge-Kutta method for numerical integration of (13), then the rotation matrices drift from the orthogonal rotation group, $\mathrm{SO}(3)$; the quantity $R^{T} R$ drifts from the identity matrix. Then the attitudes of the rigid bodies cannot be determined accurately, resulting in significant errors in the gravitational force and moment computations that depend on the attitude, and consequently errors in the entire simulation. It is often proposed to parameterize (13) by Euler angles or unit quaternions. However, Euler angles are not global expressions of the attitude since they have associated singularities. Unit quaternions do not exhibit singularities, but are constrained to lie on the unit three-sphere $\mathbb{S}^{3}$, and general numerical integration methods do not preserve the unit length constraint. Therefore, quaternions lead to the same numerical drift problem. Re-normalizing the quaternion vector at each step tends to break the conservation properties. Furthermore, unit quaternions double cover $\mathrm{SO}(3)$, so that there are inevitable ambiguities in expressing the attitude.

In contrast, the Lie Group Variational Integrator has desirable properties such as symplecticity, momentum preservation, and good energy stability for exponentially long time periods. It also preserves the Euclidian Lie group structure without the use of local charts, reprojection, or constraints. The LGVI is obtained by discretizing Hamilton's principle; the velocity phase space of the continuous Lagrangian is replaced by discrete variables, and a discrete Lagrangian is chosen such that it approximates a segment of the action integral. Taking the variation of the resulting action sum, we obtain discrete EOM referred to as a variational integrator. Since the discrete variables are updated by Lie group operations, the group structure 
is preserved. Here we present the resulting discrete EOM as follows; the detailed development can be found in Ref. 25.

$$
\begin{gathered}
X_{n+1}=F_{2_{n}}^{T}\left(X_{n}+h V_{n}-\frac{h^{2}}{2 m} \frac{\partial U_{n}}{\partial X_{n}}\right), \\
h S\left(J_{R_{n}} \Omega_{n}-\frac{h}{2} M_{n}\right)=F_{n} J_{d R_{n}}-J_{d R_{n}} F_{n}^{T}, \\
h S\left(J_{2} \Omega_{2_{n}}+\frac{h}{2} X_{n} \times \frac{\partial U_{n}}{\partial X_{n}}+\frac{h}{2} M_{n}\right)=F_{2_{n}} J_{d_{2}}-J_{d_{2}} F_{2_{n}}^{T}, \\
R_{n+1}=F_{2_{n}}^{T} F_{n} R_{n}, \\
V_{n+1}=F_{2_{n}}^{T}\left(V_{n}-\frac{h}{2 m} \frac{\partial U_{n}}{\partial X_{n}}\right)-\frac{h}{2 m} \frac{\partial U_{n+1}}{\partial X_{n+1}}, \\
J_{R_{n+1}} \Omega_{n+1}=F_{2_{n}}^{T}\left(J_{R_{n}} \Omega_{n}-\frac{h}{2} M_{n}\right)-\frac{h}{2} M_{n+1}, \\
J_{2} \Omega_{2_{n+1}}=F_{2_{n}}^{T}\left(J_{2} \Omega_{2_{n}}+\frac{h}{2} X_{n} \times \frac{\partial U_{n}}{\partial X_{n}}+\frac{h}{2} M_{n}\right)+\frac{h}{2} X_{n+1} \times \frac{\partial U_{n+1}}{\partial X_{n+1}}+\frac{h}{2} M_{n+1} .
\end{gathered}
$$

To propagate these equations, we start with a set of initial states, $\left(X_{0}, V_{0}, R_{0}, \Omega_{0}, \Omega_{2_{0}}\right)$, and perform one initial evaluation of the mutual potential gradients, obtaining $\partial U_{0} / \partial X_{0}$ and $M_{0}$ with Eqs. (3) and (17). We then find $X_{1}$ from Eq. (21). Solving the implicit equations (22) and (23) yields the matrix-multiplication update matrices $F_{0}$ and $F_{2_{0}}$ for the attitude rotation matrices, and $R_{1}$ follows from Eq. (24). After that, we use $X_{1}$ and $R_{1}$ in a new evaluation of the mutual potential gradients. We then compute $V_{1}, \Omega_{1}$, and $\Omega_{2_{1}}$ from equations (25), (26) and (27), respectively. This yields a discrete map $\left(X_{0}, V_{0}, R_{0}, \Omega_{0}, \Omega_{2_{0}}\right) \mapsto$ $\left(X_{1}, V_{1}, R_{1}, \Omega_{1}, \Omega_{2_{1}}\right)$, and this process can be repeated for each time step. Note that only one new evaluation of the potential gradients is required per time step. The discrete trajectory in reduced variables can be used to reconstruct the inertial motion of the bodies. Either concurrently with that propagation or later after completion of it, through storing values, we can use the gradient $\partial U / \partial X$, the relative attitude $R$, and the update matrix $F_{2}$ with these equations:

$$
\begin{gathered}
x_{2_{n+1}}=x_{2_{n}}+h v_{2_{n}}+\frac{h^{2}}{2 m_{2}} R_{n} \frac{\partial U_{n}}{\partial X_{n}} \\
v_{2_{n+1}}=v_{2_{n}}+\frac{h}{2 m_{2}} R_{n} \frac{\partial U_{n}}{\partial X_{n}}+\frac{h}{2 m_{2}} R_{n+1} \frac{\partial U_{n+1}}{\partial X_{n+1}} \\
R_{2_{n+1}}=R_{2_{n}} F_{2_{n}}
\end{gathered}
$$

In the discrete map defined by the LGVI above, the only implicit parts are Eqs. (22) and (23). These two equations have the same structure, which suggests a specific computational approach. Using the Rodrigues formula, we rewrite those equations as an equivalent vector equations, and we solve them numerically using Newton's iteration. Numerical simulations show that two or three iterations are sufficient to achieve a tolerance of $\epsilon=10^{-15}$.

\section{B. Properties of the Lie Group Variational Integrator}

Since the LGVI is obtained by discretizing Hamilton's principle, it is symplectic and preserves the structure of the configuration space, $\mathrm{SE}(3)$, as well as the relevant geometric features of the full two rigid body problem dynamics represented by the conserved first integrals of total angular momentum and total energy. The total energy oscillates around its initial value with small bounds on a comparatively short timescale, but there is no tendency for the mean of the oscillation in the total energy to drift (increase or decrease) from the initial value for exponentially long time. In contrast, the total energy behavior with general numerical methods such as the Runge-Kutta schemes tends to drift dramatically over exponentially long time.

The LGVI preserves the group structure. By using the given computational approach, the matrices $F_{n}$ and $F_{2_{n}}$, representing the change in relative attitude and attitude of $\mathcal{B}_{2}$ over a time step, are guaranteed to be rotation matrices. The group operation of the Lie group $\mathrm{SO}(3)$ is matrix multiplication. Hence rotation 
matrices $R_{n}$ and $R_{2_{n}}$ are updated by the group operation in Eqs. (24) and (30), so that they evolve on $\mathrm{SO}(3)$ automatically without constraints or reprojection. Therefore, the orthogonal structure of the rotation matrices is preserved, and the attitude of each rigid body is determined accurately and globally without the need to use local charts (parameterizations) such as Euler angles or quaternions.

This geometrically exact numerical integration method yields a highly efficient and accurate computational algorithm, especially for the full two rigid body problem examined here. In the full two rigid body problem there is a large burden in computing the mutual gravitational force and moment for arbitrary bodies, so the number of force and moment evaluations should be minimized. We have seen that the LGVI requires only one such evaluation per integration step, the minimum number of evaluations consistent with the presented LGVI having second order accuracy (because it is a self-adjoint method). Within the LGVI, two implicit equations must be solved at each time step to determine the matrix-multiplication updates for $R$ and $R_{2}$. However the LGVI is only weakly implicit in the sense that the iteration for each implicit equation is independent of the much more costly gravitational force and moment computation. The computational load to solve each implicit equation is comparatively negligible; only two or three iterations are required. Altogether, the entire method could be considered "almost explicit".

The LGVI is a fixed step size integrator, but all of the properties above are independent of the step size. Consequently, we can achieve the same level of accuracy while choosing a larger step size as compared to other numerical integrators of the same order. All of these features are revealed in the simulation results below.

\section{Numerical Examples}

\section{A. Implementation Details}

Here we describe our specific implementation of the combined computational methods outlined in this paper. Our codes consist of pre-processing scripts and post-processing scripts written in the MATLAB scripting language, and actual executables written in the $\mathrm{C}$ language.

Body models for a natural body or for a spacecraft are originally provided to us already in the form of ordered vertex and face lists. Each row of the vertex list contains three numbers for the coordinates of that vertex's position with respect to a specified reference frame, preferably with origin at the center of mass of the body and axes aligned with the body's principle axis. Each row of the face list contains the three row numbers for the rows of the vertex list corresponding to the three vertices that form the corners of that face. The row numbers reference the vertex information in order, moving counterclockwise around the vertices as viewed from outside of the body (faces are oriented with outward-pointing normal vectors according to the right hand rule). If enough information about density distribution for the body is known, so that a density number is also assigned to the simplex associated with each face, those numbers are present in an additional column in the ordered face list. Otherwise, all simplices may be given the same density value. In this paper we do not address the process of generating such formatted body model files from actual observation data for asteroids or from actual CAD software models for spacecraft. The face and vertex lists can be manually generated from scratch for simple arbitrary polyhedron shapes, as for the octahedra bodies used in the next section. In addition to the body models themselves, the initial conditions are needed, including the initial attitude of each body with respect to some common reference frame, the initial spin axis orientation and spin rate of each body in that frame, and the initial mutual orbital elements or equivalent relative translational motion parameters with respect to that frame.

The pre-processing scripts handle a number of preliminary computations. For each body, the centroid (center of mass) and the principal axis directions are found, and if not at the origin and along the elementary unit vectors, respectively, of the frame in which the vertex coordinates are given, the coordinates of every vertex are shifted and rotated so that this becomes the case. The moment of inertia contribution of each simplex in the new frame is found, and these are summed to obtain the total standard moment of inertia matrix. Other information such as the surface area, volume, and mean equivalent radius of each body is also found and reported. All vertex coordinates and other quantities can then optionally be nondimensionalized using user-entered length, mass, and time factors, for better numerical conditioning. Finally, five files are produced. The first two of these files, one for each body, contains a re-ordering of the elements of the position vectors, with respect to the centroid, for the vertices of the simplices. The other three files, respectively, contain the initial state vector $\left(X_{0}, m V_{0}, J_{2} \Omega_{2_{0}}, J_{R_{0}} \Omega_{0}, R_{0}, R_{2_{0}}\right)$, other system physical data (densities, body volumes, $J_{1}, J_{2}, m_{1}, m_{2}, m$, nondimensionalized $G$, and nondimensionalization factors), and the integration 
parameters (starting and stopping times and truncation error tolerance, if needed).

A first executable makes use of the Runge-Kutta-Fehlberg 7(8) integration method, hereafter referred to as RKF7(8) or just RKF, to propagate the system, after a starting calculation of the time-invariant $\mathbf{Q}$ tensors. It is noted that this one-time only calculation of the $\mathbf{Q}^{\prime} s$ is analogous to finding the successively higher-order mass moments of a "normalized" simplex with vertices at $(0,0,0),(1,0,0),(0,1,0)$, and $(0,0,1)$. It is also noted that for this high-order scheme, the EOM are evaluated thirteen times within each integration step, and an evaluation of the mutual gravitational potential force and moment is required each of those times. After this the state update is performed and the new state vector is written to an output file only if the truncation error is within the tolerance specified. Step size adjustment is performed with every step. With each state update the mutual potential itself, force, and moment, while not required for the propagation of the dynamics, are also evaluated again using the new state vector and written to another output file. This allows for checking the total energy conservation and the linear and angular accelerations. Hence fourteen force and moment evaluations are needed per integration step.

Another executable uses the LGVI. At the start of this, the initial state vector from the input file, $\left(X_{0}, m V_{0}, J_{2} \Omega_{2_{0}}, J_{R_{0}} \Omega_{0}, R_{0}, R_{2_{0}}\right)$, is converted to the vector for the discrete mapping, $\left(X_{0}, V_{0}, R_{0}, \Omega_{0}, \Omega_{2_{0}}\right)$. Again, rather than fourteen force and moment evaluations, this LGVI involves only one such evaluation per integration step, plus the quick solution of the implicit equations. Step size is fixed, but this does not present a significant problem for most scenarios observed in binary asteroids having mutual orbits with relatively low-eccentricity.

With the aim of completing simulations much faster than in a single-processor environment, a parallelized version of each executable above was also written in $\mathrm{C}$ with the addition of MPI. In using the methods presented in this paper, most of the computation time is associated with the evaluation of the potential gradients, and that involves performing the same operations for all of the different simplex combinations, followed by a global sum. This is well-suited for parallelization. The parallelized version of each executable is flexible in that any number of nodes or processors can be specified by the user. Then the process 0 assigns to each of the other processors the task of calculating the portion of the potential gradients double summations of Eqs. (3) and (6) that arises from pairing a single simplex in $\mathcal{B}_{2}$ with all simplices in $\mathcal{B}_{1}$ in succession. If the number of other processors specified by the user or found available on the cluster is less than the number of simplices in $\mathcal{B}_{2}$, this is done in rounds until the portion of the problem matching with every $a$ is obtained. The parallelized version of each executable has been used on Myrinet clusters at the Center for Advanced Computing (CAC) at the University of Michigan and at the Supercomputing and Visualization Center at NASA's Jet Propulsion Laboratory. Though compiler and user environment differences produced markedly different capabilities in each cluster environment, eventually a further two orders of magnitude reduction in computation time over otherwise identical single-processor runs was achieved with both the VI and RKF7(8) schemes. It should be noted however, that for the simulations with results presented in the following section, the parallel computing capability was not needed for such small (in number of faces) body models, and was not utilized.

The MATLAB script post-processing of the output files generates all desired plots of various dynamic quantities, with optional capability for animation generation. All pre- and post-processing steps and scripts are identical regardless of whether the RKF7(8) or LGVI executable is used, and regardless of whether the single-processor or parallel version of each is used.

\section{B. Simulation Results}

Simulation results for two octahedral rigid bodies with eight faces and eight simplices each are given for a variety of scenarios. Octahedra are used rather than more complex shapes because they are the simplest polyhedral shapes that manifest the coupled dynamics behavior desired in all of the scenarios. For greater simplicity, the octahedra are made symmetric about all axes, although they are of different sizes. The extents data defining the locations of the corners of each octahedron are given in Table 1, as are various physical parameters of each octahedron including mass and moment of inertia properties. We present simulation results for four scenarios, and the initial condition for each scenario is given in Table 2.

SCEnARIo 1 The first scenario presented here is that of short-duration simulation of the two octahedra starting from initial conditions matching with a medium eccentricity elliptical mutual orbit. Both the RKF7(8) and LGVI integrators are used, with the intent of making a direct comparison between the trajectories of the configuration variables that result from using each integrator over a short simulation duration. 
Table 1. Properties of octahedral body models used in simulations

\begin{tabular}{|c|c|c|c|c|}
\hline Property & & 2 & \multicolumn{2}{|c|}{$\mathcal{B}_{1}$} \\
\hline Surface area $\left(\mathrm{m}^{2}\right)$ & & 8.839 & & 2.002 \\
\hline Volume $\left(\mathrm{m}^{3}\right)$ & & 1.800 & & 0.1561 \\
\hline Equiv. radius (m) & & .7546 & & 0.3340 \\
\hline Mass (kg) & & 4500 & & 390.3 \\
\hline Density $\left(\mathrm{kg} / \mathrm{m}^{3}\right)$ & & 2500 & & 2500 \\
\hline$I_{x x}\left(\mathrm{~kg}-\mathrm{m}^{2}\right)$ & & 377.0 & & 9.24 \\
\hline$I_{y y}\left(\mathrm{~kg}-\mathrm{m}^{2}\right)$ & & 814.5 & & 42.99 \\
\hline$I_{z z}\left(\mathrm{~kg}-\mathrm{m}^{2}\right)$ & & 462.5 & & 44.32 \\
\hline Extents $(\mathrm{m})$ & $\min$ & $\max$ & $\min$ & $\max$ \\
\hline body frame $\mathrm{X}$ & -1.0 & 1.0 & -1.0 & 1.0 \\
\hline body frame $\mathrm{Y}$ & -1.5 & 1.5 & $-1 / \exp (1)$ & $1 / \exp (1)$ \\
\hline body frame Z & -0.9 & 0.9 & $-1 / \pi$ & $1 / \pi$ \\
\hline
\end{tabular}

Table 2. Initial Conditions

\begin{tabular}{c|l|l|c}
\hline \hline Scenario & Attitude* $(\mathrm{deg})$ Body spin $^{\dagger}(\mathrm{rad} / \mathrm{s})$ & Orbital elements $(\mathrm{m}, \mathrm{deg})$ OR state vector $(\mathrm{m}, \mathrm{m} / \mathrm{s})$ \\
\hline \multirow{2}{*}{1} & $\begin{array}{c}(100,9.8,175) \\
(160,-5,165)\end{array}$ & $\begin{array}{c}\left(0,0,5.0 \times 10^{-5}\right) \\
\left(0,0,9.2 \times 10^{-5}\right)\end{array}$ & $(a, e, i, \Omega, \omega, \nu)=\left(4 \mathrm{~m}, 0.3,5^{\circ}, 15^{\circ}, 60^{\circ}, 10^{\circ}\right)$ \\
\hline \multirow{2}{*}{2} & $\begin{array}{c}(180,0,30) \\
(270,0,30)\end{array}$ & $\begin{array}{c}(0,0,0.566) \\
(0,0,-0.566)\end{array}$ & $X_{0}=[0,6,0]^{T}, V_{0}=[-0.006,0,0]^{T}$ \\
\hline \multirow{2}{*}{3} & $\begin{array}{c}(-22.6,5,180) \\
(50.3,5,-180)\end{array}$ & $\begin{array}{l}\left(0,0,1.63 \times 10^{-4}\right) \\
\left(0,0,1.55 \times 10^{-4}\right)\end{array}$ & $(a, e, i, \Omega, \omega, \nu)=\left(52.9 \mathrm{~m}, 0.942,5^{\circ}, 0^{\circ}, 88.2^{\circ},-107.1^{\circ}\right)$ \\
\hline \multirow{2}{*}{4} & $(-75,30,180)$ & $(0.007,0.007,0.05)$ & $X_{0}=[-0.5,1.8,1.1]^{T}, V_{0}=[-0.3,-0.1,0]^{T}$ \\
\hline \hline
\end{tabular}

* 3-1-3 Euler sequence for $\mathcal{B}_{1}$ (first line) and $\mathcal{B}_{2}$ (second line).

$\dagger$ Components of angular velocity of each body expressed in its own body-fixed frame for $\mathcal{B}_{1}$ (first line) and $\mathcal{B}_{2}$ (second line).

Figure 1 shows the difference between the output of the RKF7(8) and that of the LGVI in components of reconstructed inertial position, inertial velocity, and body-frame angular velocity vectors for $\mathcal{B}_{2}$, plus the difference in body attitude parameters for $\mathcal{B}_{2}$. The corresponding output difference plots for body $\mathcal{B}_{1}$ look very similar. The differences in vector components of Figure 1(a) are normalized by the system's semi-major axis $(a=4.0 \mathrm{~m})$. The differences in vector components of Figure 1(b) are normalized by the equivalent circular velocity $\left(\sqrt{\mu / a}=2.856 \times 10^{-4} \mathrm{~m} / \mathrm{s}\right)$, and those of Figure $1(\mathrm{c})$ are normalized by the equivalent meanmotion $\left(\sqrt{\mu / a^{3}}=7.141 \times 10^{-5}\right.$ radians/s $)$. To obtain the results compared here, the total number of mutual potential derivatives evaluations and actual running time using the RKF7(8) routine were 70014 and 494 seconds, respectively, while the number of such evaluations and actual running time using the LGVI were 70001 and 539 seconds. Therefore the computational effort and resources used were roughly the same in each case. All of these results show that the LGVI can be trusted to produce almost exactly the same trajectory as a standard RKF7(8) integration routine over short time scales. As the simulation duration increases the trajectories from the two integrators begin to diverge. The behavior of integrals of motion and appropriate error metrics must then be used to discern which trajectory is to be taken as the "truth".

SCEnARIo 2 Another scenario is that of propagation from an initial condition with the bodies aligned but possessing relatively large magnitude centroid velocity vectors that are antiparallel and perpendicular to the initial line between centroids. This scenario is simulated both with the LGVI at different step sizes and 


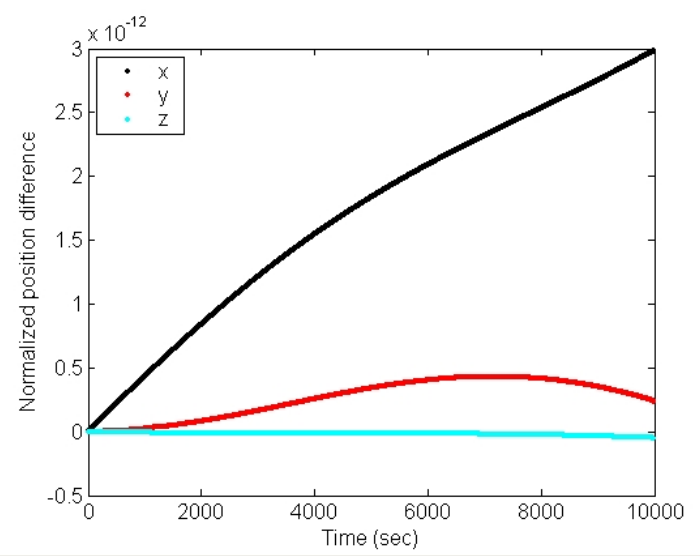

(a) Inertial position for $\mathcal{B}_{2}$

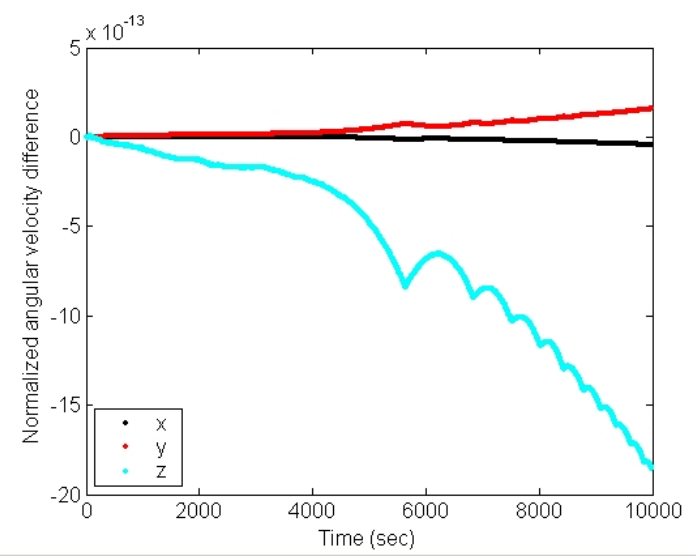

(c) Angular velocity for $\mathcal{B}_{2}$

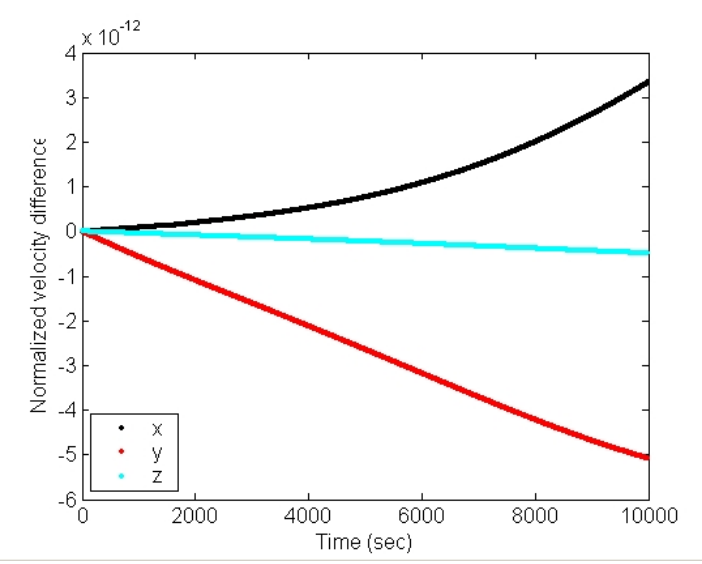

(b) Inertial velocity for $\mathcal{B}_{2}$

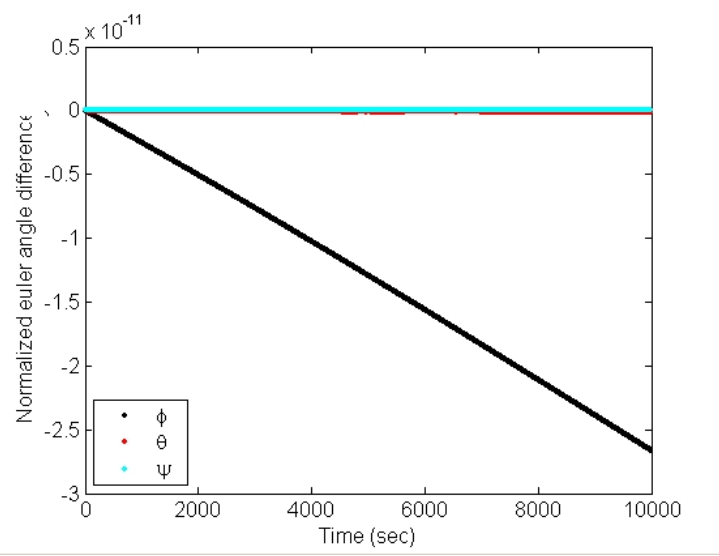

(d) 3-1-3 Euler angles for $\mathcal{B}_{2}$

Figure 1. (Scenario 1) Difference between RKF7(8) and LGVI output.

with the variable step size RKF7(8) at different error tolerances. This allows for a comparison between the integrators of their performance, in terms of the total energy and total angular momentum integrals and the attitude error metric growth vs. computational burden. The results in Table 3 illustrate the general superiority of the LGVI approach over Runge-Kutta-type approaches.

Here we see that for any pair of simulations, one using the LGVI and the other using the RKF7(8) scheme, for which the total energy metric performs about the same, the computation time needed to complete the simulation using the LGVI is a fraction of that needed using the RKF7(8). Simultaneous with this improvement in run time, the total angular momentum and attitude error metrics still perform better in the LGVI run than in the RKF7(8) run by multiple orders of magnitude. Going in the other direction, as the step size for the LGVI is reduced so that the computational burden using it begins to approach that for any chosen run using the RKF7(8), all error metrics remain at the same level as or else orders of magnitude smaller than those for the chosen RKF7(8) run. For the LGVI, the round-off error accumulates when multiplying rotation matrices at (24). The rotation matrix error of the LGVI is caused only by the floating-point arithmetic operation, and it is increased as the number of integration steps is increased. A similar trend is observed in the total angular momentum error for the LGVI, because determination of the total angular momentum in the inertial frame from the states written to the output file makes use of the rotation matrices.

SCEnARIo 3 The next scenario illustrates the ability of our methods to capture the interesting effects of coupling in a mutual orbit configuration that the Keplerian two-body approximation incorrectly predicts as being perpetual. Simulation with the LGVI yields the trajectory illustrated in Figure 2(a), which transitions from a highly elliptical orbit to a hyperbolic escape path. This is shown by the plots in Figure 2(b) of the 
Table 3. (Scenario 2) Performance comparison between RKF7(8) and LGVI

\begin{tabular}{l|cccc|ccc}
\hline \hline Method & $h^{*}$ & $N_{u}^{\star}$ & $t_{W}^{\diamond}$ & $\epsilon^{\triangleleft}$ & $\mathrm{E}[|\Delta \mathrm{TE}|]^{\dagger \ddagger}$ & $\mathrm{E}\left[\left\|\Delta \pi_{T}\right\|\right]^{\dagger \ddagger}$ & $\mathrm{E}\left[\left\|I-R^{T} R\right\|\right]^{\dagger}$ \\
\hline RKF7(8) & 0.236 & 2368912 & 23439 & $10^{-12}$ & $3.901 \times 10^{-12}$ & $1.493 \times 10^{-9}$ & $1.151 \times 10^{-7}$ \\
RKF7(8) & 0.421 & 1331414 & 9102 & $10^{-10}$ & $1.274 \times 10^{-10}$ & $2.630 \times 10^{-7}$ & $1.985 \times 10^{-5}$ \\
RKF7(8) & 0.749 & 747376 & 5252 & $10^{-8}$ & $2.284 \times 10^{-8}$ & $4.620 \times 10^{-5}$ & $3.173 \times 10^{-3}$ \\
\hline LGVI & 0.0169 & 2370000 & 13511 & - & $1.698 \times 10^{-11}$ & $5.167 \times 10^{-10}$ & $2.525 \times 10^{-11}$ \\
LGVI & 0.04 & 1000000 & 9920 & - & $1.928 \times 10^{-11}$ & $1.189 \times 10^{-10}$ & $2.120 \times 10^{-11}$ \\
LGVI & 0.08 & 500000 & 5127 & - & $9.879 \times 10^{-11}$ & $4.139 \times 10^{-11}$ & $2.004 \times 10^{-12}$ \\
LGVI & 0.4 & 100000 & 983 & - & $2.234 \times 10^{-9}$ & $6.266 \times 10^{-12}$ & $3.386 \times 10^{-14}$ \\
LGVI & 0.8 & 50000 & 431 & - & $9.326 \times 10^{-9}$ & $1.279 \times 10^{-11}$ & $6.352 \times 10^{-14}$ \\
LGVI & 1.0 & 40000 & 335 & - & $1.512 \times 10^{-8}$ & $3.991 \times 10^{-12}$ & $4.786 \times 10^{-14}$ \\
\hline \hline
\end{tabular}

${ }^{*} h$ is integration step size, in seconds, fixed for LGVI but averaged over the run's duration for RKF7(8)

${ }^{\star} N_{u}$ is the total number of calculations of the mutual potential derivatives made within the run

${ }^{\diamond} t_{W}$ is the "wall-clock" time to complete each simulation run, in seconds

$\triangleleft \epsilon$ is the error tolerance for the variable step size in RKF7(8)

$\ddagger \mathrm{TE}$ and $\pi_{T}$ are total energy and the total angular momentum, respectively, while $\Delta$ refers to deviation from the initial value over simulation

${ }^{\dagger} \mathrm{E}[\cdot]$ denotes mean

semi-major axis and eccentricity change during the close encounter, which occurs roughly midway through the run duration of 60,000 seconds. The initial conditions and body configurations are symmetric about the initial orbital plane, and as such the motion of the centroids should be restricted to the initial orbital plane. This is observed numerically, as the body centroids remain within $8.6 \times 10^{-14}$ meters of the initial orbital plane throughout the simulation.

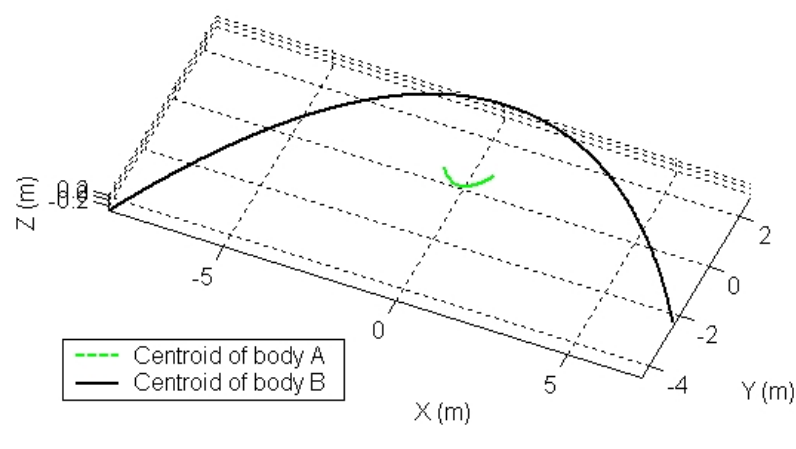

(a) Trajectory of binary octahedra system components
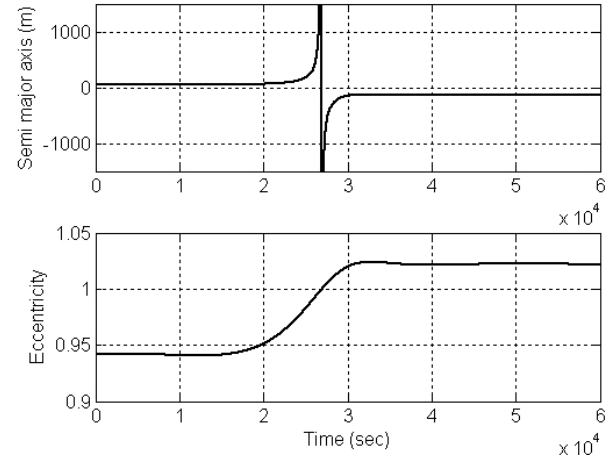

(b) Eccentricity and semi major axis

Figure 2. (Scenario 3) Disruption of the binary octahedra system.

SCEnARIo 4 Finally, we examine a very long duration simulation starting from initial conditions that are stable in the sense that orbital trajectories are confined to separated and bounded regions but are highly unstable in the sense that body attitudes vary greatly and irregularly. The trajectory of binary octahedra system is shown in Figure 3. Note that this motion cannot be observed with the point mass assumption in the classical two body problem. This scenario is simulated both with LGVI at different step sizes and with RKF7(8) at different error tolerances, for $5 \times 10^{6}(\mathrm{sec})$ maneuver time. Table 4 illustrates the general superiority of the LGVI approach, similar to Scenario 2. In addition, we see that the simulation results of RKF7(8) with larger error tolerances, $10^{-13}$ and $10^{-10}$, are completely unreliable since the rotation matrix error is increased to the unacceptable levels of $10^{-1}$ and $10^{1}$.

The quantitative comparison is summarized in Figure 4, where the total computation time and the stan- 


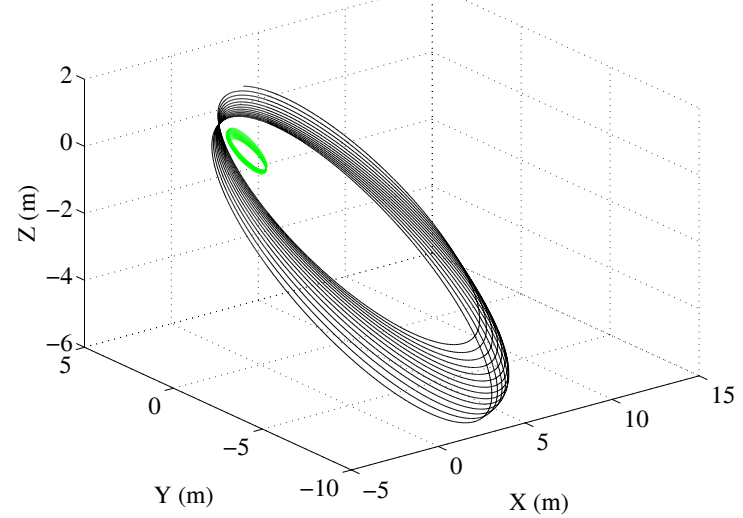

Figure 3. (Scenario 4) Trajectory of binary octahedra system

Table 4. (Scenario 4) Performance comparison between RKF7(8) and LGVI

\begin{tabular}{l|cccc|ccc}
\hline \hline Method & $h$ & $N_{u}$ & $t_{W}$ & $\epsilon$ & $\mathrm{E}[|\Delta \mathrm{TE}|]$ & $\mathrm{E}\left[\left\|\Delta \pi_{T}\right\|\right]$ & $\mathrm{E}\left[\left\|I-R^{T} R\right\|\right]$ \\
\hline RKF7(8) & 8.81 & 7373106 & 48850 & $10^{-16}$ & $6.198 \times 10^{-8}$ & $3.830 \times 10^{-6}$ & $6.089 \times 10^{-5}$ \\
RKF7(8) & 20.66 & 3145532 & 20873 & $10^{-13}$ & $8.731 \times 10^{-5}$ & $7.427 \times 10^{-3}$ & $1.015 \times 10^{-1}$ \\
RKF7(8) & 48.74 & 1333475 & 9699 & $10^{-10}$ & $1.959 \times 10^{-1}$ & $5.246 \times 10^{0}$ & $1.834 \times 10^{1}$ \\
\hline LGVI & 0.7 & 7142858 & 46963 & - & $6.947 \times 10^{-11}$ & $2.190 \times 10^{-12}$ & $6.645 \times 10^{-13}$ \\
LGVI & 2 & 2500000 & 17320 & - & $5.687 \times 10^{-10}$ & $5.077 \times 10^{-13}$ & $3.663 \times 10^{-13}$ \\
LGVI & 5 & 1000000 & 6897 & - & $3.601 \times 10^{-9}$ & $8.447 \times 10^{-13}$ & $1.642 \times 10^{-13}$ \\
LGVI & 10 & 500000 & 3750 & - & $1.517 \times 10^{-8}$ & $2.567 \times 10^{-13}$ & $3.521 \times 10^{-13}$ \\
\hline \hline
\end{tabular}

See Table 3 for notations.

dard deviation of the total energy are shown over the number of the mutual potential derivatives calculations. In Figure 4(a), we see that the total computation time is directly proportional to the number of the mutual potential derivative calculations regardless of the integration scheme used. This justifies the statement that the LGVI is an "almost explicit" computational method; the computational load to solve the implicit equations is comparatively negligible. Considering that the mutual potential derivative computations are the major computational burden for the numerical simulation of full two rigid body dynamics, an efficient integration scheme with fewer mutual potential derivative calculations should exhibit smaller error measures. In Figure 4(b), the lower left portion represents higher computational efficiency, and the upper right portion represents lower efficiency. We see that LGVI is more efficient than RKF7(8). Figure 5 compares the total energy deviation history and the relative rotation matrix error history. For LGVI, the total energy varies within the level of $10^{-11}$. The repeated peaks of the total energy deviation correspond to the perigees of the orbit shown in Figure 3, but but there is no tendency for the mean of the variation to drift for the entire simulation time. The rotation matrix error is slightly increasing, since the round off error in multiplying orthogornal matrices at (24) is accumulated. But the error is below $10^{-12}$ over the entire simulation's span. However, for RKF7(8), the variation of the total energy is linearly increasing over time, and the rotation matrix error is increasing to the level of $10^{-4}$. We have seen that LGVI exhibits good total energy behavior for exponentially long time periods, and it also preserves the group structure well. The accuracy of RKF7(8) is vulnerable to a long time simulation of the full two rigid body dynamics.

\section{Conclusions}

This paper presents a complementary combination of tools or algorithms that achieves superior performance in the computationally demanding simulation of the full two rigid bodies problem: a simple method 


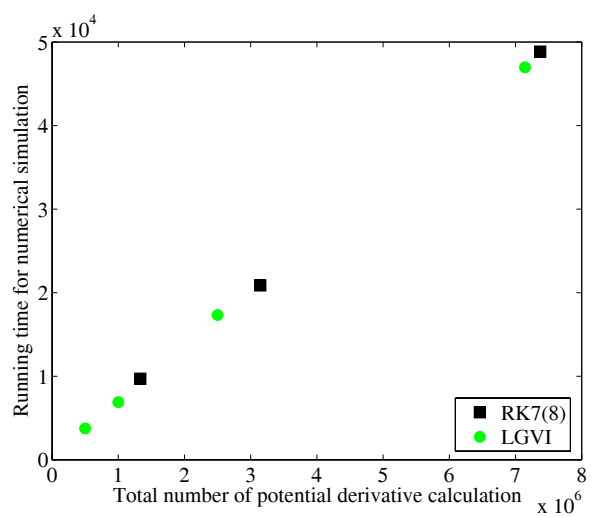

(a) Running time v.s. number mutual potential derivatives calculations

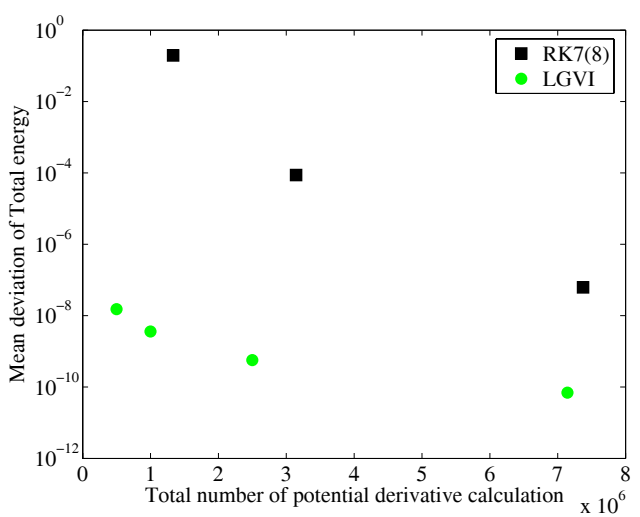

(b) Mean deviation of TE v.s. number of mutual potential derivatives calculations

Figure 4. (Scenario 4) Quantitative comparisons of running time and standard deviation of total energy over number of mutual potential derivatives calculations.
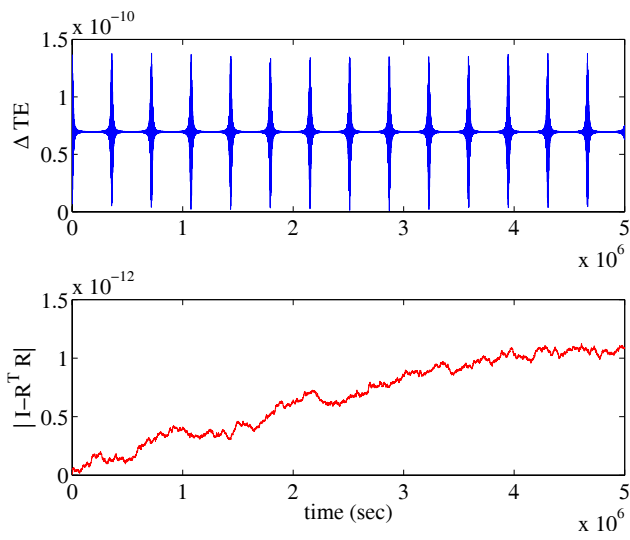

(a) LGVI
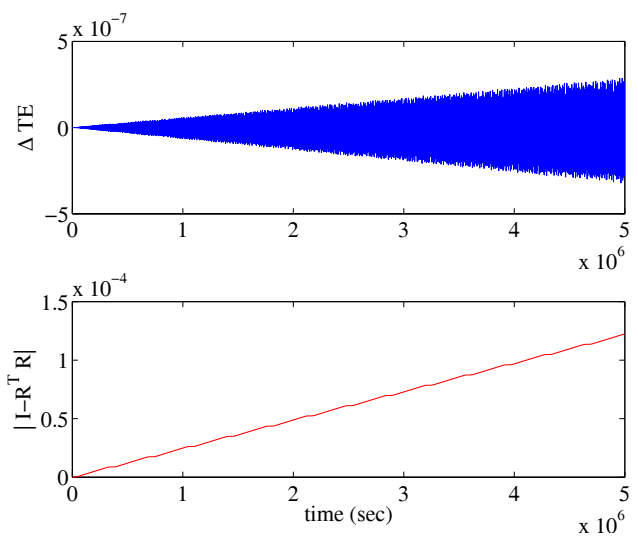

(b) $\operatorname{RKF} 7(8)$

Figure 5. (Scenario 4) Qualitative comparisons of behavior of deviation in total energy and rotation matrix error over time with similar computational load for LGVI and RKF7(8).

for efficient calculation of the mutual gravitational potential and its derivatives given versatile polyhedral models of the rigid bodies and a Lie group variational integrator consisting of discrete relative equations of motion that preserves the geometric features and structure of the configuration space. The use of these easily implemented methods together allows for simulation of the full two rigid bodies, capturing their fully coupled dynamics, that is both accurate and efficient. The results above show the accuracy maintained for energy, momenta, and attitude geometry constraints, even for long simulation run times. The presented mutual potential computations for polyhedral approximations are themselves efficient, but for bodies of relevant (i.e. large) model size, they still represent the bulk of the computational burden during simulation, regardless of the integration scheme used. Hence use of the Lie Group Variational Integrator, which requires minimal mutual potential computations, achieves an even greater combined efficiency. This yields maximal simulation durations for fixed computational resources.

\section{Acknowledgments}

The first author would like to acknowledge the support of the U. S. Air Force Office of Scientific Research (AFOSR) for the period in which this paper was written. The research of the third author was partially supported by NSF grant DMS-0504747 and a University of Michigan Rackham faculty grant. 


\section{References}

${ }^{1}$ Werner, R. A. and Scheeres, D. J., "Mutual Potential of Homogenous Polyhedra," Celestial Mechanics and Dynamical Astronomy, Vol. 91, No. 3, March 2005, pp. 337-349.

${ }^{2}$ Bottke, W. F. and Melosh, H. J., "The Formation of Binary Asteroids and Doublet Craters," Icarus, Vol. 124, 1996, pp. 372391 .

${ }^{3}$ Bottke, W. F. and Melosh, H. J., "The Formation of Asteroid Satellites and Doublet Craters by Planetary Tidal Forces," Nature, Vol. 381, 1996, pp. 5153.

${ }^{4}$ Margot, J. L., Nolan, M. C., Benner, L. A. M., Ostro, S. J., Jurgens, R. F., Giorgini, J. D., Slade, M. A., and Campbell, D. B., "Binary Asteroids in the Near-Earth Object Population," Science, Vol. 296, May 2002, pp. 1445-1448.

${ }^{5}$ Merline, W. J., Weidenschilling, S. J., Durda, D. D., Margot, J. L., Pravec, P., and Storrs, A. D., "Asteroids do have satellites," Asteroids III, edited by W. F. Bottke et al., Space Science Series, University of Arizona, Tuscon, AZ, 2002, pp. $289-312$.

${ }^{6}$ Ostro, S. J., Hudson, R. S., Benner, L. A. M., Giorgini, J. D., Magri, C., Margot, J.-L., and Nolan, M. C., "Asteroid Radar Astronomy," Asteroids III, edited by W. F. Bottke et al., Space Science Series, University of Arizona, Tuscon, AZ, 2002, pp. 151-168.

${ }^{7}$ Maciejewski, A. J., "Reduction, Relative Equilibria and Potential in the Two Rigid Bodies Problem," Celestial Mechanics and Dynamical Astronomy, Vol. 63, No. 1, 1995, pp. 1-28.

${ }^{8}$ Scheeres, D. J., "Stability in the Full Two-Body Problem," Celestial Mechanics and Dynamical Astronomy, Vol. 83, 2002, pp. 155-169.

${ }^{9}$ Scheeres, D. J., "Stability of Relative equilibria in the Full Two-Body Problem," New Trends in Astrodynamics Conference, Jan. 2003.

${ }^{10}$ Scheeres, D. J. and Augenstein, S., "Spacecraft motion about binary asteroids," Proc. AAS/AIAA Astrodynamics Specialist Conference, Aug. 2003.

${ }^{11}$ Gabern, F., Koon, W. S., and Marsden, J. E., "Spacecraft dynamics near a binary asteroid," Proceedings of the fifth international conference on dynamical systems and differential equations, Jun 2004.

${ }^{12}$ Scheeres, D. J. and Bellerose, J., "The Restricted Hill Full 4-Body Problem: application to spacecraft motion about binary asteroids," Dynamical Systems: An International Journal, Vol. 20, No. 1, 2005, pp. 23-44.

${ }^{13}$ Borderies, N., "Mutual gravitational potential of N solid bodies," Celestial Mechanics, Vol. 18, No. 3, 1978 , pp. $295-307$.

${ }^{14}$ Braun, C. V., The gravitational potential of two arbitrary, rotating bodies with applications to the Earth-Moon system, Ph.D. thesis, University of Texas at Austin, 1991.

${ }^{15}$ Moritz, H., Advanced Physical Geodesy, Abacus Press, 1980.

${ }^{16}$ Geissler, P., Petit, J.-M., Durda, D. D., Greenberg, R., Bottke, W., Nolan, M., and Moore, J., "Erosion and Ejecta Reaccretion of 243 Ida and Its Moon," Icarus, Vol. 120, No. 1, 1996, pp. 140-157.

${ }^{17}$ Ashenberg, J., "Proposed Method for Modeling the Gravitational Interaction Between Finite Bodies," Journal of Guidance, Control, and Dynamics, Vol. 28, No. 4, 2005, pp. 768-774.

${ }^{18}$ Werner, R. A. and Scheeres, D. J., "Exterior Gravitation of a Polyhedron Derived and Compared with Harmonic and Mascon Gravitation Representations of Asteroid 4769 Castalia," Celestial Mechanics and Dynamical Astronomy, Vol. 65, No. 3, 1997, pp. 313-344.

${ }^{19}$ Fahnestock, E. G., Scheeres, D. J., McClamroch, N. H., and Werner, R. A., "Simulation and Analysis of Binary Asteroid Dynamics Using Mutual Potential and Potential Derivatives Formulation," Proc. AAS/AIAA Astrodynamics Specialist Conference, Lake Tahoe, CA, Aug. 2005.

${ }^{20}$ Fahnestock, E. G. and Scheeres, D. J., "Simulation of the Full Two Rigid Body Problem Using Polyhedral Mutual Potential and Potential Derivatives Approach," Celestial Mechanics and Dynamical Astronomy, submitted for publication.

${ }^{21}$ Hairer, E., Lubich, C., and Wanner, G., Geometric Numerical Integration, Springer, 2000.

${ }^{22}$ Marsden, J. E. and West, M., "Discrete mechanics and variational integrators," Acta Numerica, Vol. 10, 2001, pp. 357514 .

${ }^{23}$ Iserles, A., Munthe-Kaas, H. Z., Nørsett, S. P., and Zanna, A., "Lie-group methods," Acta Numerica, Vol. 9, 2000, pp. 215-365.

${ }^{24}$ Lee, T., Leok, M., and McClamroch, N. H., "A Lie group variational integrator for the attitude dynamics of a rigid body with application to the 3D pendulum," Proceedings of the IEEE Conference on Control Application, Toronto, Canada, Aug. 2005, pp. 962-967.

${ }^{25}$ Lee, T., Leok, M., and McClamroch, N. H., "Lie group variational integrators for the Full Body Problem," Computer Methods in Applied Mechanics and Engineering, submitted, Available: http://arxiv.org/abs/math.NA/0508365.

${ }^{26}$ Yeomans, D. K. et al., "Radio Science Results During the NEAR-Shoemaker Spacecraft Rendezvous with Eros," Science, Vol. 289, Sept. 2000, pp. 2085-2088.

${ }^{27}$ Fujiwara, A., Kawaguchi, J., Yeomans, D. K., Abe, M., Mukai, T., Okada, T., Saito, J., Yano, H., Yoshikawa, M., Scheeres, D. J., Barnouin-Jha, O., Cheng, A. F., Demura, H., Gaskell, R. W., Hirata, N., Ikeda, H., Kominato, T., Miyamoto, H., Nakamura, A. M., Nakamura, R., Sasaki, S., , and Uesugi, K., "The Rubble-Pile Asteroid Itokawa as Observed by Hayabusa," Science, accepted for publication.

${ }^{28}$ Richardson, D. C., William F. Bottke, J., and Love, S. G., "Tidal Distortion and Disruption of Earth-Crossing Asteroids," Icarus, Vol. 134, 1998, pp. 47-76.

${ }^{29}$ Roig, F., Duffard, R., Penteado, P., Lazzaro, D., and Kodama, T., "Interacting ellipsoids: a minimal model for the dynamics of rubble-pile bodies," Icarus, Vol. 165, No. 2, 2003, pp. 355-370.

${ }^{30}$ Korycansky, D. G., "Orbital Dynamics for Rigid Bodies," Astrophysics and Space Science, Vol. 291, 2004, pp. 57-74.

${ }^{31}$ Duan, Z.-H. and Krasny, R., "An Adaptive Treecode for Computing Nonbonded Potential Energy in Classical Molecular Systems," Journal of Computational Chemistry, Vol. 22, No. 2, 2001, pp. 184-195. 